\title{
Prediction of Acute in vivo Toxicity of Some Amine and Amide Drugs to Rats by Multiple Linear Regression, Partial Least Squares and an Artificial Neural Network
}

\author{
Mohamad Khayatzadeh MAHANI,* Marzieh ChaloosI,*† Mohamad Ghanadi MaragheH,** \\ Ali Reza KhanCHI, ** and Daryoush AfZaLI*** \\ * Faculty of Chemistry, Tarbiat Moalem University, 49 Mofateh Avenue, Tehran, Iran \\ **Jaber Ibn Hayan Research Laboratories, Nuclear Science and Technology Research Institute, AEOI, \\ P. O. Box 11365-8486, Tehran, Iran \\ ***Faculty of Science, Department of Chemistry, Shahid Bahonar University, Kerman, Iran
}

\begin{abstract}
The oral acute in vivo toxicity of 32 amine and amide drugs was related to their structural-dependent properties. Genetic algorithm-partial least-squares and stepwise variable selection was applied to select of meaningful descriptors. Multiple linear regression (MLR), artificial neural network (ANN) and partial least square (PLS) models were created with selected descriptors. The predictive ability of all three models was evaluated and compared on a set of five drugs, which were not used in modeling steps. Average errors of 0.168, 0.169 and 0.259 were obtained for MLR, ANN and PLS, respectively.
\end{abstract}

(Received February 14, 2007; Accepted March 15, 2007; Published September 10, 2007)

\section{Introduction}

One of the current interests in medicinal chemistry and toxicology is the classification of chemical substances with respect to their toxicity toward living systems. ${ }^{1}$ A large number of time-consuming and costly in vivo animal tests are still required to assess the toxicity of a drug substance. Computers and statistical software now make it possible to move conventional animal evaluation programs away from unavoidable experimental and biological variables onto a more objective plane where physicochemical properties and chemical reactivity can be evaluated individually as factors determining events. ${ }^{2}$ Several methods have emerged for the evaluation and prediction of the biological effects of chemicals. One of the methods that have shown greatest promise is the use of quantitative structural-activity relationships (QSAR). ${ }^{3-7}$ QSAR have provided a valuable tool in research on the toxicity of organic chemicals, which is based on the premise that the toxicity of a chemical is related to the physico-chemical structure; thus, toxicity can be related to the ability of a compound to reach the site of action, and its ability to react covalently there. ${ }^{8}$ Such phenomena can be modeled adequately using physico-chemical parameters, such as hydrophobicity and electrophilicity. ${ }^{9,10}$ Juranic et al. ${ }^{1}$ have developed multiple linear regression (MLR) and partial least squares regression (PLS) models for predicting the oral acute toxicity of some Nsubstituted fluoroacetamides toward rats. These QSAR models have the advantage that they can predict toxicity, but only normally for very a restricted series of compounds.

In this study, a molecular modeling study was performed for

$\dagger$ To whom correspondence should be addressed.

E-mail: mchaloosi@gmail.com the optimization of the 3-D molecular structures of a group of 32 amine and amide drugs. Also, the oral acute in vivo toxicity of these drugs towards rats was correlated with their structure related properties. The aim of this work is to create, optimize and evaluate MLR, artificial neural network (ANN) and PLS models for predicting the toxicity of drugs as an alternative to animal experimentation.

\section{Experimental}

\section{Software and methods}

Stepwise variable selection, MLR and PLS algorithms were written in MATLAB Ver. 6.5 (MathWorks, Inc., Natick, MA, USA). GA-PLS variable selection algorithms were used from PLS-Toolbox for MATLAB Ver. 2.0 (Eigenvector Research Group, Inc., Manson, WA, USA) with some corrections. Hyperchem for windows Ver. 7.0 (Hypercube, Inc., Alberta, Canada) and Dragon Ver. 3.0 (Milano Chemometrics and QSAR Research Group, Milano, Italy) were used for optimizing the molecular structures and calculating the molecular descriptors, respectively. All programs were run on a PC Pentium IV with the Windows XP operating system.

\section{Data set and descriptors}

The median lethal dose $\left(\mathrm{LD}_{50}\right)$ was used for evaluating the acute toxicity. $\mathrm{LD}_{50}$ is the amount of a chemical that is lethal to one-half of the experimental animals exposed to it. $\mathrm{LD}_{50}$ values are usually expressed as the amount of the chemical (mole or milligram) per unit of body weight ( $\mathrm{mol} / \mathrm{kg}$ or $\mathrm{mg} / \mathrm{kg}$ ), and may be oral (fed), dermal (applied to the skin), or inhalation (administered in the form of vapors). The oral $\mathrm{LD}_{50}$ to rat of 32 amine and amide drugs was used to estimate acute in vivo toxicity (Table 1). The data set used in this research was 
Table 1 Oral acute in vivo toxicity of drugs to rat $\left(\mathrm{LD}_{50}\right)$

\begin{tabular}{llll}
\hline \multicolumn{1}{c}{ Compound } & $\begin{array}{c}\text { Oral LD } 50 \text { to } \\
\text { rat/mol kg-1 }\end{array}$ & Compound & $\begin{array}{l}\text { Oral LD } \mathrm{LD}_{50} \text { to } \\
\mathrm{rat} / \mathrm{mol} \mathrm{kg}^{-1}\end{array}$ \\
\hline $\begin{array}{l}\text { Acetaminophen } \\
\text { Amethopterin }\end{array}$ & 0.0156910 & Indomethacin & 0.0010850 \\
Amitriptyline & 0.0003000 & Iproniazid & 0.0000341 \\
Antipyrine & 0.0095600 & Milrinone & 0.0032700 \\
$\quad$ phenazone) & 0.0019300 & Mitoxantrone & 0.0000419 \\
Azathioprine & 0.0012400 & Natulan & 0.0013200 \\
Carbaryl & 0.0002500 & Orphenadrine & 0.0035410 \\
Chlorambucil & 0.0105000 & Papaverine & 0.0015780 \\
Chloramphenicol & 0.0182000 & Phenacetin & 0.0009600 \\
Chlorhexidine & 0.0004550 & Phenobarbital & 0.0092100 \\
Chlorpromazine & 0.0014260 & Phenylephrine & 0.0006970 \\
Codeine & 0.0024900 & Piperazine & 0.0020900 \\
Diazepam & 0.0000535 & Scopolamine & 0.0221000 \\
Fentanyl & 0.0014610 & Tetracycline 5 & 0.0041800 \\
Fluoxetine & 0.0027600 & Verapamil & 0.0144990 \\
Glutethimide & 0.0156910 & & \\
Imipramine & & & \\
\hline
\end{tabular}

selected from the Registry of Cytotoxicity (RC) database. HyperChem entered the structures of molecules to a computer. Before calculating the molecular descriptors, optimization of the 3D molecular structures was performed by a semi-empirical AM1 method. Dragon calculated 1242 none-zero descriptors (constitutional, topological, properties etc.). Thirteen descriptors were calculated by HyperChem. The selection of principal descriptors that relate acute in vivo toxicity to physicochemical properties or molecular structures is a main procedure in any modeling method.

\section{Variable selection}

It is well known that collinearity of the descriptors can seriously affect the stability and quality of the models. The variance inflation factor (VIF) was used for determining and evaluating the collinearity in the data set. The VIF for variable $i$ is defined as ${ }^{11}$

$$
\operatorname{VIF}(i)=\frac{1}{\left(1-R^{2}(i)\right)}
$$

where $R^{2}(i)$ is the coefficient of multiple determination for the regression between descriptor $i$ and remaining descriptors. The values of VIF of 5 (correspond to values of $R^{2}$ of 0.8 ) was assumed to be large. ${ }^{12}$ Descriptors with large values of VIF were removed. After this step, 820 descriptors were rejected and 435 descriptors remained. Because this large number of descriptors leads to time-consuming calculations and invalid models, variable selection methods were applied to the dataset. Two procedures that included genetic algorithm (GA) and stepwise procedures were used.

The genetic algorithm ${ }^{13-17}$ is inspired by biological evolution. When GA applies to a subset selection problem, we need a regression method for extracting a relationship between selected descriptors and dependent variables. Many multiple or multivariate regression methods, such as MLR, principle component regression (PCR) and PLS, can be used for this purpose. In this research, GA-PLS was used for variable selection. At the beginning, a population of 128 chromosomes, with each chromosome containing 435 zero or one element, is randomly created. Ten-percent of each chromosome elements was set to 1 at the beginning in a random selection compliant
Table 2 Selected descriptors by stepwise variable selection and GA-PLS

\begin{tabular}{|c|c|c|c|}
\hline Name & Stepwise & GA-PLS & Description \\
\hline$\Delta H_{\mathrm{f}}$ & - & $\checkmark$ & Heat of formation \\
\hline $\mathrm{HE}$ & - & $\checkmark$ & Hydration energy \\
\hline Hy & - & $\checkmark$ & Hydrophilic factor \\
\hline $\log \mathrm{P}$ & - & $\checkmark$ & $\begin{array}{l}\text { Partition coefficient between } \\
\text { octanol-water }\end{array}$ \\
\hline LUMO & - & $\checkmark$ & $\begin{array}{l}\text { Lowest unoccupied molecular } \\
\text { orbital energy }\end{array}$ \\
\hline Mor27p & $\checkmark$ & $\checkmark$ & $\begin{array}{l}\text { 3D Morse descriptor weighted by } \\
\text { atomic polarizability }\end{array}$ \\
\hline$M_{\mathrm{w}}$ & - & $\checkmark$ & Molecular weight \\
\hline O-058 & $\checkmark$ & $\checkmark$ & Number of $=\mathrm{O}$ groups \\
\hline PSA & $\checkmark$ & $\checkmark$ & Polar surface area \\
\hline$R$ & - & $\checkmark$ & Refractivity \\
\hline
\end{tabular}

with a uniform distribution across the descriptor range. If the $i$ th element of chromosome is one, then the $i$ th descriptor is selected; if zero, then the $i$ th descriptor is rejected. With each chromosome, a model is created by PLS, and the predicted error sum of squares (PRESS) is calculated by leave one out cross validation. At the next step, two different chromosomes are selected from the population as parents. From the crossover between these chromosomes (mating), two children are created, which are evaluated by a regression method. If the children with the minimum PRESS are better than the worst member of the population, then it is substituted. During mating between the population members, mutation takes place at a 0.005 rate. This procedure repeats 150 times; at the end, descriptors that are applied by the chromosome with the minimum PRESS were selected. After GA variable selection, 10 descriptors are selected. We carried out this procedure. The descriptors with a short description are given in Table 2 .

Stepwise variable selection ${ }^{18}$ is a combination of forward and backward variable selection. At the beginning in a forward selection procedure, an MLR model was constructed with a descriptor that had highest correlation with $\mathrm{LD}_{50}$, and then the partial $F$ was calculated for all other descriptors according to the following equation:

$$
F=\frac{\mathrm{SS}_{\mathrm{reg}(\mathrm{new})}-\mathrm{SS}_{\mathrm{reg}(\mathrm{old})}}{\mathrm{MS}_{\mathrm{res}(\mathrm{new})}} .
$$

Here, $\mathrm{SS}_{\text {reg(new) }}$ and $\mathrm{SS}_{\text {reg(old) }}$ are the regression sum of squares for the model with and without a new descriptor, and $\mathrm{MS}_{\text {res(new) }}$ is the residual mean squares for the model with a new descriptor. The descriptor with the highest partial $F$ was entered into the model. After this step in a backward elimination procedure, the partial $F$ was calculated for each variable in the model, as though it was added last to the model. The descriptor that did not significantly contribute to the regression equation was rejected. These procedures were repeated until no descriptor was entered to or removed from the model at a defined probability. Stepwise selection is a simple and fast procedure, but usually it is not used to make a selection among more than 20 descriptors. For this purpose, the stepwise variable selection was applied to selected descriptors by GA. Three descriptors were selected, and are shown in Table 2.

\section{Modeling methods}

Artificial neural network. ANNs consist of layers that contain small units, called neurons. Each neuron contains input, 
weights associated with each input, transfer function and output. Weights are considered to be distributed knowledge content of the network. ANNs "learn" from examples, and exhibit some capability to generalize beyond the training data. Such a model bears great similarity to a nervous system. Many different types of ANNs have been developed according to learning algorithms for adjusting weigths. Back propagation (BP), or multilayer feed forward (MLF) networks, ${ }^{22}$ were used in this research. ANNs can be applied for linear and non-linear calibration and modeling. The ANN model is defined by the number of neurons (nodes) in the input, hidden and output layers and weights associated with each interconnection and input. The number of descriptor or independent variables and predicted or dependent variables determines the number of nodes in the input and output layers, respectively. ${ }^{23}$

A large number of input variables in ANN modeling cause increased training time, memory storage, and reduced generalization ability and robustness of the model. To overcome these problems, different methods, such as increasing the number of samples, decreasing the number of descriptors by different variable selection procedures and compressing the input data by such techniques as principle-component analysis are proposed. In this work, decreasing the number of descriptors by variable selection was used to surmount the problems.

Partial least squares. The PLS ${ }^{19}$ method takes into account information of dependent variables during the decomposition of the independent variables data matrix. A non-linear iterative partial least squares (NIPALS) algorithm ${ }^{20}$ was used in the present work. With this algorithm, the data matrix of the descriptors is compressed to a smaller data matrix, called "scores", in a new coordinate system. The new coordinate axes are called latent variables (LVs), or factors, and are linear combinations of the original variables. A LV represents a systematic variation found in the data set. The regression coefficients from each original variable to each LV are called "loadings". PLS assumes the dependent variable to be a linear combination of scores. ${ }^{21}$

The number of LVs to be used within the PLS algorithm is an important parameter to achieve better performance in prediction. This allows modeling of the system with the optimum amount of information and the avoidance of overfitting or under-fitting. The leave-one-out and segmented crossvalidation procedures consist of systematically removing one or a group of the training samples in turn, and using only the remaining ones for constructing the model. In our study, the predicted values were then compared with the actual ones for a calibration set, and PRESS was calculated. PRESS was computed in the same manner, and each time a new factor was added to the PLS model.

$$
\operatorname{PRESS}=\sum_{i=1}^{n}\left(A_{\mathrm{i}}-P_{\mathrm{i}}\right)^{2}
$$

Here $A_{\mathrm{i}}$ is the actual value and $P_{\mathrm{i}}$ is the predicted value for sample $i$, and $n$ is the number of samples.

\section{Results and Discussion}

The oral $\mathrm{LD}_{50}$ to rat of selected drugs are given in Table 1. The data set, consisting of 32 drugs, was divided into two data sets (calibration and prediction) by ranking based on the $\mathrm{LD}_{50}$ values. In this splitting method, the diversity in dependent variable is taken into account, but the structural similarity is not
Table 3 Descriptive parameters of MLR, ANN and PLS models

\begin{tabular}{|c|c|c|c|c|}
\hline \multirow{2}{*}{ Descriptor } & \multirow{2}{*}{$\frac{\text { MLR }}{\begin{array}{l}\text { Regression } \\
\text { coefficient }\end{array}}$} & \multirow{2}{*}{$\begin{array}{c}\text { ANN } \\
\begin{array}{c}\text { Weight in } \\
\text { hidden layer }\end{array}\end{array}$} & \multicolumn{2}{|c|}{ PLS } \\
\hline & & & $\begin{array}{l}\text { Loading } \\
\text { on LV }\end{array}$ & $\begin{array}{l}\text { Regression } \\
\text { coefficient }\end{array}$ \\
\hline Constant & 1.5725 & $-0.1356^{\mathrm{a}}$ & - & - \\
\hline$\Delta H_{\mathrm{f}}$ & - & - & -0.06368 & -0.0548 \\
\hline HE & - & - & 0.03243 & 0.2068 \\
\hline Hy & - & - & -0.21976 & -0.3026 \\
\hline $\log P$ & - & - & 0.03817 & 0.0303 \\
\hline LUMO & - & - & -0.45515 & -0.3213 \\
\hline Mor27p & -3.1500 & -0.3350 & -0.35328 & -0.5519 \\
\hline$M_{\mathrm{w}}$ & - & - & 0.54644 & 0.3341 \\
\hline O-058 & 0.3995 & 0.2299 & 0.23046 & 0.3521 \\
\hline PSA & 0.0075 & 0.1109 & 0.35594 & 0.2719 \\
\hline$R$ & - & - & 0.58007 & 0.3800 \\
\hline
\end{tabular}

a. Weight associated with bias neuron in hidden layer.

considered. Our calibration and prediction sets contained 27 and 5 samples, respectively.

\section{MLR and ANN models}

The MLR analysis technique was employed for relating the oral acute toxicity of drugs to selected descriptors. Selected variables by a stepwise procedure ( 3 variables) were subjected to MLR analysis. The regression coefficients of the MLR model are given in Table 3. A cross-validated correlation coefficient $\left(Q^{2}\right)$ of 0.85 was obtained for this model. The influence of each descriptor is described by the regression coefficient. In this model, the 3D Morse descriptor that is weighted by atomic polarizability (Mor27p) is a principal descriptor, and is related to the molecular bulk or the molecular volume. ${ }^{24}$ The negative sign of this descriptor indicates that increasing it leads to a lower drug toxicity (high values of $\mathrm{LD}_{50}$ ). Two other descriptors, containing a number of double bonds to an oxygen atom (O-058), a functional group parameter and the polar surface area (PSA) as an electronic parameter are needed for a full description of acute toxicity. As expected from the positive sign of these two descriptors, the toxicity was increased by increasing these parameters.

In order to compare the predictive ability of MLR with ANN, the dataset was modeled by ANN. An artificial neural network with sigmoid and linear transfer functions after hidden and output layers, respectively was trained by a back-propagation algorithm. The network weights initially assigned random values between +1 and -1 . The best performance of the network was obtained with 1 neuron in a hidden layer. Because there are three descriptors and one independent variable in the data set, the input and output layers consisted of 3 and 1 neuron, respectively. Thus, a 3-1-1 network was used. In Table 3, the weights associated with each descriptor in the hidden layer's neuron are given. In this model, Mor27p has the highest weight, and is principal descriptor and the weights of O-058 and PSA reduce, respectively, as in MLR model. This correlation between two models supports same mechanism as the MLR model.

From Table 4, it is clearly observed that the correlations between the descriptors that were used in the MLR and ANN models are low. MLR and ANN were applied to a prediction set, which contained samples that were not use in any previous calibrations. Table 5 gives these predicted values and their absolute errors. The results show that, with the same number of 
Table 4 Intercorrelation matrix for descriptors that were used in MLR, ANN and PLS

\begin{tabular}{|c|c|c|c|c|c|c|c|c|c|c|}
\hline & $\Delta H_{\mathrm{f}}$ & $\mathrm{HE}$ & Нy & $\log P$ & LUMO & Mor27p & $M_{\mathrm{w}}$ & O-058 & PSA & $R$ \\
\hline$\Delta H_{\mathrm{f}}$ & 1 & 0.358 & -0.191 & 0.486 & 0.116 & -0.198 & -0.251 & -0.503 & 0.076 & -0.112 \\
\hline $\mathrm{HE}$ & & 1 & -0.641 & 0.517 & 0.436 & -0.588 & -0.551 & -0.393 & -0.546 & -0.362 \\
\hline $\mathrm{Hy}$ & & & 1 & -0.303 & -0.099 & 0.529 & 0.394 & 0.267 & 0.404 & 0.247 \\
\hline $\log P$ & & & & 1 & 0.255 & -0.264 & -0.111 & -0.406 & -0.177 & 0.017 \\
\hline LUMO & & & & & 1 & -1.140 & -0.473 & -0.400 & -0.570 & -0.352 \\
\hline Mor27p & & & & & & 1 & 0.045 & 0.479 & 0.343 & -0.158 \\
\hline$M_{\mathrm{w}}$ & & & & & & & 1 & 0.259 & 0.608 & 0.951 \\
\hline O-058 & & & & & & & & 1 & 0.429 & 0.093 \\
\hline PSA & & & & & & & & & 1 & 0.439 \\
\hline$R$ & & & & & & & & & & 1 \\
\hline
\end{tabular}

Table 5 Actual values of acute in vivo toxicity and predicted values by MLR, ANN and PLS and their absolute errors

\begin{tabular}{lccrrrrr}
\hline \multicolumn{1}{c}{ Name } & Actual value, $\log \left(1 / \mathrm{LD}_{50}\right)$ & MLR $^{\mathrm{a}}$ & Error & ANN $^{\mathrm{b}}$ & Error & PLS $^{\mathrm{c}}$ & Error $^{2}$ \\
\hline Carbaryl & 2.907 & 2.717 & -0.190 & 2.697 & -0.210 & 2.565 & -0.342 \\
Fluoxetine & 2.835 & 2.930 & 0.095 & 2.919 & 0.084 & 3.216 & 0.381 \\
Glutethimide & 2.559 & 2.693 & 0.134 & 2.684 & 0.125 & 2.408 & -0.151 \\
Mefnamic acid & 2.485 & 2.774 & 0.289 & 2.763 & 0.278 & 2.712 & 0.227 \\
Natulan & 2.451 & 2.317 & -0.134 & 2.303 & -0.148 & 2.259 & -0.192 \\
Average error & - & - & 0.168 & - & 0.169 & - & 0.259 \\
\hline
\end{tabular}

a. MLR model was constructed with 3 descriptors. b. A 3-1-1 ANN model with sigmoid and linear transfer function after hidden and output layers, respectively. c. PLS model with 10 descriptors and 1 latent variables.

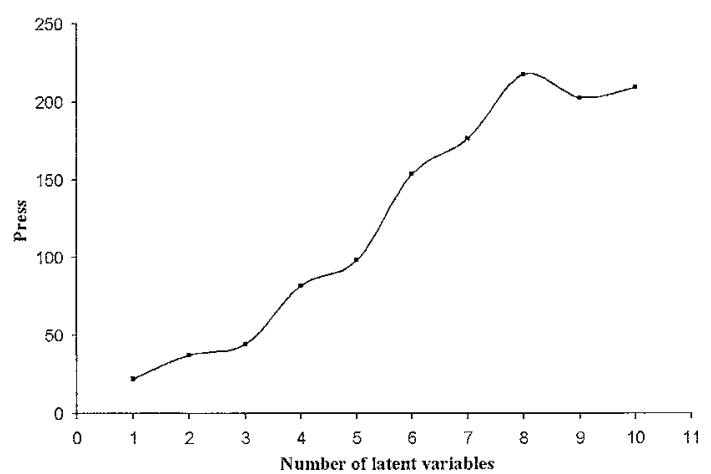

Fig. 1 Press vs. number of latent variables for the PLS model.

descriptors, the absolute errors of two methods are comparable, and MLR was preferred due to simplicity. Thus, the constructed models are applicable for predicting drug toxicity with good precision.

\section{PLS model}

For the calibration step in PLS, all 10 descriptors in Table 2 were selected (the results of PLS with 3 descriptors were not acceptable, and are not shown). After applying the PLSNIPALS algorithm to the calibration set, press was calculated, and is shown in Fig. 1. One latent variable was selected for PLS modeling due to the minimum PRESS. The loading of each descriptor on the latent variable and the regression coefficient of the PLS model are given in Table 3. The regression coefficients describe the influence of each descriptor in the model. In this model, as in the MLR model Mor27p is a principal descriptor with a negative effect on the acute toxicity. In addition, both O-058 and PSA have a positive effect on the acute toxicity. Another important parameter in the PLS model is the refractivity, when the weight is nearly the same as O-058. The negative sign of the coefficient of the enthalpy of formation $\left(\Delta H_{\mathrm{f}}\right)$ and the hydrophilic factor $(\mathrm{Hy})$ indicates that toxic drugs have low values of the hydrophilic factor and the enthalpy of formation. The hydration energy and the logarithm of the partition coefficient between octanol-water, which describe the hydrophobicity of the structure, ${ }^{9}$ have opposite effects. These results are supported by Ragno et al. ${ }^{25}$ and Hemmateenejad et al. ${ }^{26}$ in the modeling of anti-tubercular activity, Cronin et al. ${ }^{10}$ in the modeling of toxicity for 63 aliphatic compounds and Juranic et al. ${ }^{1}$ in the modeling of acute toxicity for nineteen $\mathrm{N}$-substituted fluoroacetamides. The lowest unoccupied molecular orbital (LUMO) energy describes the electrophilicity of drugs. ${ }^{9,10}$ A higher LUMO energy, which results from the occupation of a higher energy orbital, might lower the electronelectron repulsion, which results in a lower molecular energy. The important role of the molecular energy in describing the mechanism of the interaction at the molecular level in a biological system has also been previously reported. ${ }^{27}$ The negative sign of this descriptor in the PLS model indicates that toxic drugs have low values of the LUMO energy, and is according to the work of Cronin et al. ${ }^{10}$ Presumably, with increasing molecular weight $\left(M_{\mathrm{w}}\right)$ the volume and polarizability of the molecule increase, and this descriptor is needed for a full description of acute toxicity.

The correlations between all of these parameters are given in Table 4. It is clearly observed that the correlation between the descriptors is low. Table 5 gives the result of applying this PLS model to the prediction set. The results of the constructed PLS model are comparable with MLR and ANN, and this model is applicable for predicting of drug toxicity.

\section{Conclusions}

In this research, three models for the oral acute toxicity of 27 
amine and amide drugs were constructed by the MLR, ANN and PLS methods. The performances of the models were evaluated by a new set of drugs containing 5 sample. The results of these models were acceptable, but the MLR model was better, due to simplicity in the modeling step. By developing these models, it is possible to predict the oral acute toxicity as a replacement of time-consuming experiments on animals.

\section{References}

1. I. O. Juranic, B. J. Drakulic, S. D. Petrovic, D. Z. Mijin, and M. V. Stankovic, Chemosphere, 2006, 62, 641.

2. J. J. Hostynek and P. S. Magee, Toxicol. in Vitro, 1997, 11, 377.

3. H. Ertepinar, Y. Gok, O. Geban, and S. Ozden, Eur. J. Med. Chem., 1995, 30, 171.

4. C. Hansch, D. Hoekman, and H. Gao, Chem. Rev., 1996, 96, 1045.

5. C. Hansch, A. Kurup, R. Garg, and H. Gao, Chem. Rev., 2001, 101, 619.

6. P. K. Larser, T. Lilijefor, and U. Madsen, "Textbook of Drug Design \& Discovery", 2002, Taylor \& Fransis, London.

7. B. Hemmateenejad, M. Akhond, R. Miri, and M. Shamsipur, J. Chem. Inf. Comput. Sci., 2003, 43, 1328.

8. M. T. D. Cronin, IL Farmaco, 2001, 56, 149.

9. M. T. D. Cronin, J. C. Dearden, J. C. Duffy, R. Edwards, N. Manga, A. P. Worth, and A. D. P. Worgan, SAR QSAR Environ. Res., 2002, 13, 167.

10. M. T. D. Cronin, G. S. Bowers, G. D. Sinks, and T. W. Schultz, SAR QSAR Environ. Res., 2000, 11, 301.

11. D. A. Belsy, E. Kuh, and R. E. Welsch, "Regression Diagnostics-Identifying Influential Data and Sources of
Collinearity", 1980, John Wiley and Sons, NY

12. R. D. Snee, Technometrics, 1977, 19, 415.

13. C. B. Lucasius and G. Kateman, Chemom. Intell. Lab. Syst., 1993, 19,1 .

14. C. B. Lucasius and G. Kateman, Chemom. Intell. Lab. Syst., 1994, 25, 99

15. D. B. Hibbert, Chemom. Intell. Lab. Syst., 1993, 19, 277.

16. R. Leardi, R. Boggia, and M. Terrile, J. Chemom., 1992, 6, 267.

17. R. Leardi, J. Chemom., 1994, 8, 65.

18. L. Xu and W. J. Zhang, Anal. Chim. Acta, 2001, 446, 477.

19. H. Martens and T. Naes, "Multivariate Calibration", 1991, John Wiley \& Sons Ltd., Chichester.

20. M. Otto, "Chemometrics: Statistics and Computer Application in Analytical Chemistry", 1999, Wiley-VCH Verlag GmbH, Weinheim.

21. B. G. M. Vandeginste, D. L. Massart, L. M. C. Buydens, S D. Jong, P. J. Lewi, and J. S. Verbeke, "Handbook of Chemometrics and Qualimetrics Part B", ed. B. G. M. Vandeginste and A. C. Rutan, 1998, Vol. 20B, Elsevier, Amsterdam.

22. M. Hagan, H. Demuth, and M. Beale, "Neural Network Design", 1996, PWS Publishing Company, Boston.

23. J. Zupan and J. Gasteiger, "Neural Networks for Chemists", 1993, Wiley/VCH, Weinheim.

24. M. Karelson, V. S. Lobanov, and A. R. Katritzky, Chem. Rev., 1996, 96, 1027.

25. R. Ragno, G. R. Marshall, R. D. Santo, R. Costi, S. Massa, R. Rompei, and M. Artico, Bioorg. Med. Chem., 2000, 8, 1423.

26. B. Hemmateenejad, R. Miri, M. Jafarpour, M. Tabarzad, and A. Foroumadi, QSAR Comb. Sci., 2005, 25, 56.

27. V. Klimesova, K. Palat, K. Waisser, and J. Klimes, Int. J. Pharm., 2000, 207, 1. 